International Journal of Engineering, Science and Technology

Vol. 3, No. 2, 2011, pp. 146-156
INTERNATIONAL

JOURNAL OF

ENGINEERING,

SCIENCE AND

TECHNOLOGY

www.ijest-ng.com

(C) 2011 MultiCraft Limited. All rights reserved

\title{
Steady hydromagnetic Couette flow in a rotating system with non- conducting walls
}

\author{
G. S. Seth* and J. K. Singh \\ Department of Applied Mathematics, Indian School of Mines, Dhanbad-826004, INDIA \\ *Corresponding Author: e-mail: gsseth_ism@yahoo.com, Tel. +91-326-2235421, Fax. +91-326-2296619
}

\begin{abstract}
Steady hydromagnetic Couette flow of class-II of a viscous incompressible electrically conducting fluid in a rotating system with non-conducting walls is studied. Exact solution of the governing equations is obtained in closed form. Expressions for the shear stress at the lower and upper plates due to primary and secondary flows and mass flow rates in primary and secondary flow directions are also derived. Asymptotic behavior of the solution for fluid velocity and induced magnetic field is analyzed for small and large values of rotation parameter $K^{2}$ and magnetic parameter $M^{2}$ to gain further insight into the flow pattern. Heat transfer characteristics of the flow are considered taking viscous and Joule dissipations into account. The numerical solution of energy equation and numerical values of rate of heat transfer at both plates are obtained with the help of MATLAB software. The numerical values of fluid velocity, induced magnetic field and fluid temperature are depicted graphically versus channel width variable $\eta$ for various values of $M^{2}$ and $K^{2}$ while numerical values of primary and secondary shear stress at the lower and upper plates, mass flow rates in primary and secondary flow directions and rate of heat transfer at the lower and upper plates are presented in tabular form for various values of $K^{2}$ and $M^{2}$.
\end{abstract}

Keywords: Magnetic field, rotation, hydromagnetic Ekman boundary layer, Hartmann boundary layer, viscous and Joule dissipations.

\section{Introduction}

An investigation of MHD flow of an electrically conducting fluid in a rotating medium is of considerable significance due to occurrence of various natural phenomena related to astrophysical and geophysical problems and for its application in fluid engineering such as vertex MHD power generator (Michiyoshi and Numano 1967), rotating drum type separator in closed cycle two phase power generator (Lenzo at.el 1978), isotope separation and plasma diagnostics (Thiagarajan and Rohatgi 1977). Keeping in view this fact Jana et.al (1977), Seth and Maiti (1982), Jana and Datta (1980), Mandal et.al (1982), Mandal and Mandal (1983), Seth and Ahmad (1985) and Kumar et.al (2007) studied steady MHD Couette flow of a viscous incompressible electrically conducting fluid in a rotating system considering different aspects of the problem.

It may be noted that MHD Couette flow may be generated into two ways and may be named as Couette flow of class-I and classII i.e Couette flow induced due to a moving plate bounded by a stationary plate fixed at a finite distance from the moving plate may be named as MHD Couette flow of class-I which is similar to the fluid flow induced due to the movement of the plate bounded by stationary free stream whereas MHD flow past a stationary plate induced due to movement of the plate present at a finite distance from the stationary plate may be recognized as MHD Couette flow of class-II which is similar to the fluid flow past a stationary plate due to moving free stream. The research studies of Jana et.al (1977), Seth and Maiti (1982), Jana and Datta (1980), Mandal et.al (1982), Mandal and Mandal (1983), Seth and Ahmad (1985) and Kumar et.al (2007) belong to MHD Couette flow of class-I. Majumder (1991), Ganapathy (1994) and Das et.al (2008) investigated Couette flow of class-II of a viscous incompressible fluid in a rotating system in the absence of magnetic field whereas Singh (2000), Hayat et.al (2004a,b,c) and Seth et.al (2009) studied MHD Couette flow of class-II of a viscous incompressible electrically conducting fluid in the presence of a uniform magnetic field neglecting induced magnetic field by considering different aspects of the problem. It may be noted that 
induced magnetic field produced by fluid motion is negligible in comparison to the applied one when magnetic Reynolds number is very small. Although for the problems of astrophysical and geophysical interest magnetic Reynolds number is not very small. For such type of problems induced magnetic field plays a significant role in determining flow features of the problem.

The aim of the present investigation is to study steady magnetohydrodynamic Couette flow of class-II of a viscous incompressible electrically conducting fluid in a rotating system in the presence of a uniform transverse magnetic field taking induced magnetic field into account.

\section{Formulation of the problem and its solution}

Consider steady Couette flow of a viscous incompressible electrically conducting fluid confined within a parallel plate channel $y=0$ and $y=d$ with non-conducting walls in the presence of a uniform transverse magnetic field $H_{0}$ acting parallel to $y$-axis.

Both the fluid and channel rotate in unison with the uniform angular velocity $\Omega$ about $y$-axis. The fluid flow within the channel is induced due to the movement of the upper plate $y=d$ with uniform velocity $U_{0}$ in $x$-direction where as the lower plate of the channel $y=0$ is kept fixed. Since plates of the channel are infinite along $x$ and $z$-directions and flow is steady so all the physical quantities, expect pressure, depend on y only.

Therefore, fluid velocity $\vec{q}$ and induced magnetic field $\vec{H}$ are assumed as

$$
\vec{q} \equiv\left(u^{*}, 0, w^{*}\right) \text { and } \vec{H} \equiv\left(H_{x}^{*}, H_{0}, H_{z}^{*}\right) \text {, }
$$

which is compatible with the fundamental equations of Magnetohydrodynamics in a rotating frame of reference.

Taking into account the assumptions made above, the governing equations for steady flow of a viscous incompressible electrically conducting fluid in a rotating frame of reference are

$$
\begin{aligned}
& -\frac{1}{\rho} \frac{\partial p^{*}}{\partial x}+v \frac{d^{2} u^{*}}{d y^{2}}+\frac{\mu_{e} H_{0}}{\rho} \frac{d H_{x}^{*}}{d y}=2 \Omega w^{*}, \\
& -\frac{1}{\rho} \frac{\partial p^{*}}{\partial y}=0, \\
& -\frac{1}{\rho} \frac{\partial p^{*}}{\partial z}+v \frac{d^{2} w^{*}}{d y^{2}}+\frac{\mu_{e} H_{0}}{\rho} \frac{d H_{z}^{*}}{d y}=-2 \Omega u^{*}, \\
& H_{0} \frac{d u^{*}}{d y}+\eta_{m} \frac{d^{2} H_{x}^{*}}{d y^{2}}=0, \\
& H_{0} \frac{d w^{*}}{d y}+\eta_{m} \frac{d^{2} H_{z}^{*}}{d y^{2}}=0,
\end{aligned}
$$

where $\eta_{m}=1 / \mu_{e} \sigma$ and $\rho, v, \mu_{e}, \sigma$ and $p^{*}$ are, respectively, fluid density, kinematic coefficient of viscosity, magnetic permeability, electrical conductivity of the fluid and modified pressure including centrifugal force.

The formulation of the problem will be complete if we specify boundary conditions for the velocity and induced magnetic field. The boundary conditions for the velocity and induced magnetic field are given by

$$
\begin{array}{ll}
u^{*}=w^{*}=0 & \text { at } y=0, \\
u^{*}=U_{0}, w^{*}=0 & \text { at } y=d, \\
H_{X}{ }^{*}=H_{Z}{ }^{*}=0 & \text { at } y=0, \\
H_{x}{ }^{*}=H_{z}{ }^{*}=0 & \text { at } y=d .
\end{array}
$$

Equation (3) shows constancy of magnetohydrodynamic pressure along $y$-axis i.e. along the axis of rotation. Keeping into account the research studies made in the past on MHD Couette flow we are of opinion that MHD Couette flow may be induced in two ways viz. (i) Couette flow induced due to a moving plate bounded by a stationary plate fixed at a finite distance from the moving plate may be named as MHD Couette flow of class-I which is similar to the fluid flow induced due to the movement of the plate bounded by stationary free stream and (ii) MHD flow past a stationary plate induced due to movement of the plate present at a finite distance from the stationary plate may be recognized as MHD Couette flow of class-II which is similar to the fluid flow past a stationary plate due to moving free stream. For MHD Couette flow of class-I the pressure gradient terms $-\frac{1}{\rho} \frac{\partial p^{*}}{\partial x}$ and 
$-\frac{1}{\rho} \frac{\partial p^{*}}{\partial z}$; which are present in equations (2) and (4), are not considered by Jana et.al (1977), Seth and Maiti (1982), Jana and

Datta (1980), Mandal et.al (1982), Mandal and Mandal (1983), Seth and Ahmad (1985) and Kumar et.al (2007). This is justified and is clearly evident from the conditions (7a) and (8a). For MHD Couette flow of class-II the pressure gradient terms in equations (2) and (4) are obtained with the help of boundary conditions (7b) and (8b) which are given by

$$
-\frac{1}{\rho} \frac{\partial p^{*}}{\partial x}=0,-\frac{1}{\rho} \frac{\partial p^{*}}{\partial z}=-2 \Omega U_{0} .
$$

Our problem belongs to MHD Couette flow of class-II therefore, equations (2) and (4) with the help of (9) reduce to

$$
\begin{aligned}
& v \frac{d^{2} u^{*}}{d y^{2}}+\frac{\mu_{e} H_{0}}{\rho} \frac{d H_{x}{ }^{*}}{d y}=2 \Omega w^{*}, \\
& v \frac{d^{2} w^{*}}{d y^{2}}+\frac{\mu_{e} H_{0}}{\rho} \frac{d H_{z}{ }^{*}}{d y}=-2 \Omega\left(u^{*}-U_{0}\right),
\end{aligned}
$$

We introduce the non-dimensional variables

$$
\eta=y / d, u=u^{*} / U_{0}, w=w^{*} / U_{0}, H_{x}=H_{x}^{*} / H_{0}, H_{z}=H_{z}^{*} / H_{0} .
$$

Equations (5), (6), (10) and (11) with the use of (12), in non-dimensional form, become

$$
\begin{aligned}
& \frac{d u}{d \eta}+\frac{1}{R_{m}} \frac{d^{2} H_{x}}{d \eta^{2}}=0, \\
& \frac{d w}{d \eta}+\frac{1}{R_{m}} \frac{d^{2} H_{z}}{d \eta^{2}}=0, \\
& \frac{d^{2} u}{d \eta^{2}}+\frac{M^{2}}{R_{m}} \frac{d H_{x}}{d \eta}=2 K^{2} w, \\
& \frac{d^{2} w}{d \eta^{2}}+\frac{M^{2}}{R_{m}} \frac{d H_{z}}{d \eta}=2 K^{2}-2 K^{2} u,
\end{aligned}
$$

where $K^{2}=\Omega d^{2} / v$ is rotation parameter which is reciprocal of Ekman number, $M^{2}=\mu_{e}{ }^{2} H_{0}{ }^{2} d^{2}(\sigma / \rho v)$ is magnetic parameter which is square of Hartmann number and $R_{m}=U_{0} d / \eta_{m}$ is magnetic Reynolds number.

The boundary conditions ( $7 \mathrm{a})$ to $(8 \mathrm{~b})$, in non-dimensional form, become

$$
\begin{array}{ll}
u=w=0 & \text { at } \eta=0, \\
u=1, \quad w=0 & \text { at } \eta=1, \\
H_{X}=H_{z}=0 & \text { at } \eta=0, \\
H_{X}=H_{z}=0 & \text { at } \eta=1 .
\end{array}
$$

Combining equations (13) and (15) with the equations (14) and (16) respectively, we obtain

$$
\begin{aligned}
& \frac{d F}{d \eta}+\frac{d^{2} b}{d \eta^{2}}=0, \\
& \frac{d^{2} F}{d \eta^{2}}+M^{2} \frac{d b}{d \eta}+2 i K^{2} F=2 i K^{2},
\end{aligned}
$$

where $F=u+i w, b=h_{x}+i h_{z}, h_{x}=H_{x} / R_{m}$ and $h_{z}=H_{z} / R_{m}$.

The boundary conditions (17a) to (18b) become

$$
\begin{aligned}
& F=0 \text { at } \eta=0 \text { and } F=1 \text { at } \eta=1, \\
& b=0 \text { at } \eta=0 \quad \text { and } b=0 \text { at } \eta=1 .
\end{aligned}
$$

Equations (19) and (20) subject to the boundary conditions (21) and (22) are solved and the solution for velocity field and induced magnetic field is expressed as

$$
\begin{aligned}
& F(\eta)=[A \sinh (\lambda \eta)-B(1-\cosh (\lambda \eta))], \\
& b(\eta)=\frac{1}{\lambda}\left[A(1-\cosh (\lambda \eta))-B \sinh (\lambda \eta)+\lambda(B+1) \frac{2 i K^{2} \eta}{M^{2}}\right],
\end{aligned}
$$


where $\lambda=\alpha-i \beta$,

$$
\begin{aligned}
& \alpha, \beta=\frac{1}{\sqrt{2}}\left[\left(M^{4}+4 K^{4}\right)^{1 / 2} \pm M^{2}\right]^{1 / 2}, \\
& A=\frac{1}{2}\left[\frac{2 i \lambda K^{2} \cosh \lambda-M^{2} \sinh \lambda}{i \lambda K^{2} \sinh \lambda+M^{2}(1-\cosh \lambda)}\right], \\
& B=-\frac{1}{2}\left[\frac{2 i \lambda K^{2} \sinh \lambda+M^{2}(1-\cosh \lambda)}{i \lambda K^{2} \sinh \lambda+M^{2}(1-\cosh \lambda)}\right] .
\end{aligned}
$$

\section{Shear Stress at plates}

The non-dimensional shear stress components $\tau_{X}$ and $\tau_{Z}$ at the lower and upper plates due to primary and secondary flows respectively are given by

$$
\begin{aligned}
& \left(\tau_{x}+i \tau_{z}\right)_{\eta=0}=\frac{\lambda}{2}\left[\frac{2 i \lambda K^{2} \cosh \lambda-M^{2} \sinh \lambda}{i \lambda K^{2} \sinh \lambda+M^{2}(1-\cosh \lambda)}\right], \\
& \left(\tau_{x}+i \tau_{z}\right)_{\eta=1}=\frac{\lambda}{2}\left[\frac{2 i \lambda K^{2}-M^{2} \sinh \lambda}{i \lambda K^{2} \sinh \lambda+M^{2}(1-\cosh \lambda)}\right] .
\end{aligned}
$$

\section{Mass flow rates}

The non-dimensional mass flow rates $Q_{X}$ and $Q_{Z}$ in the primary and secondary flow directions respectively are given by

$$
Q_{x}+i Q_{z}=\frac{2 i \lambda K^{2}-\lambda B}{M^{2}} \text {. }
$$

\section{Asymptotic Solutions}

We shall now discuss asymptotic behavior of the solution given by (23) to (25) for small and large values of $M^{2}$ and $K^{2}$ to gain some physical insight into the flow pattern.

Case I: $M^{2}<<1$ and $K^{2}<<1$

Since $M^{2}$ and $K^{2}$ are very small, neglecting squares and higher powers of $M^{2}$ and $K^{2}$ in equations (23) to (25), we obtain velocity and induced magnetic field from (23) to (25) as

$$
\begin{aligned}
& u=\eta+\frac{M^{2}}{12} \eta\left(2 \eta^{2}-3 \eta+1\right)+\cdots, \\
& w=-\frac{K^{2}}{3} \eta\left(\eta^{2}-3 \eta+2\right)+\cdots, \\
& h_{x}=\frac{1}{2} \eta(1-\eta)\left[1-\frac{M^{2}}{12} \eta(1-\eta)\right]+\cdots, \\
& h_{z}=\frac{K^{2}}{12} \eta(1-\eta)\left(1+3 \eta-\eta^{2}\right)+\cdots .
\end{aligned}
$$

It is evident from the expressions (29) to (32) that in a slowly rotating system when the conductivity of the fluid is low and/ or the applied magnetic field is weak, primary velocity $u$ and primary induced magnetic field $h_{X}$ are independent of rotation where as secondary velocity $w$ and secondary induced magnetic field $h_{z}$ are unaffected by magnetic field.

Case II: $K^{2}>>1$ and $M^{2} \sim O(1)$

When $K^{2}$ is large and $M^{2}$ is of small order of magnitude flow becomes boundary layer type. For boundary layer flow near the lower plate $\eta=0$, we obtain velocity and induced magnetic field from equations (23) to (25) as 


$$
\begin{aligned}
& u=1-e^{-\alpha_{1} \eta} \cos \beta_{1} \eta, \\
& w=-e^{-\alpha_{1} \eta} \sin \beta_{1} \eta, \\
& h_{x}=\frac{1}{2 K}\left[(1-\eta)-e^{-\alpha_{1} \eta}\left(\cos \beta_{1} \eta-\sin \beta_{1} \eta\right)\right], \\
& h_{z}=\frac{1}{2 K}\left[(1-\eta)-e^{-\alpha_{1} \eta}\left(\cos \beta_{1} \eta+\sin \beta_{1} \eta\right)\right],
\end{aligned}
$$

where

$$
\alpha_{1}=K\left(1+\frac{M^{2}}{4 K^{2}}\right) \text { and } \beta_{1}=K\left(1-\frac{M^{2}}{4 K^{2}}\right) .
$$

Equations (33) to (37) demonstrate the existence of a boundary layer of thickness of $O\left(\alpha_{1}^{-1}\right)$ near lower plate of the channel. This boundary layer may be identified as hydromagnetic Ekman boundary layer and can be viewed as classical Ekman boundary layer modified by magnetic field. The thickness of this boundary layer decreases with the increase in either $M^{2}$ or $K^{2}$. Exponential terms in equations (33) to (36) damped quickly as $\eta$ increases. When $\eta>1 / \alpha_{1}$ i.e. outside the boundary layer region, we obtain from (33) to (36) as

$$
\begin{aligned}
& u \approx 1, w \approx 0, \\
& h_{x} \approx(1-\eta) / 2 K, h_{z} \approx(1-\eta) / 2 K .
\end{aligned}
$$

It is noticed from (38) and (39) that in a certain core given by $\eta>1 / \alpha_{1}$ i.e. outside the boundary layer region, fluid flows in primary flow direction only with the same velocity as that of moving plate. Primary and secondary induced magnetic fields $h_{X}$ and $h_{Z}$ persist and decrease on increasing channel width variable $\eta$.

Case III: $\quad M^{2}>>1$ and $K^{2} \sim O(1)$

In this case also boundary layer type flow is expected. For the boundary layer flow near the lower plate $\eta=0$, we obtain velocity and induced magnetic field from the equations (23) to (25) as

$$
\begin{aligned}
& u=\frac{1}{2}\left[1-e^{-\alpha_{2} \eta}\left\{\cos \beta_{2} \eta+\frac{K^{2}}{M} \sin \beta_{2} \eta\right\},\right. \\
& w=-\frac{1}{2}\left[\frac{K^{2}}{M}+e^{-\alpha_{2} \eta}\left\{\sin \beta_{2} \eta-\frac{K^{2}}{M} \cos \beta_{2} \eta\right\}\right], \\
& h_{x}=\frac{1}{2 M}\left\{1-e^{-\alpha_{2} \eta}\left(\cos \beta_{2} \eta+\frac{K^{2}}{M} \sin \beta_{2} \eta\right)\right\} \text {, } \\
& h_{z}=\frac{1}{2 M}\left\{\frac{K^{2}}{M}(2 \eta-1)-e^{-\alpha_{2} \eta}\left(\sin \beta_{2} \eta-\frac{K^{2}}{M} \cos \beta_{2} \eta\right)\right\} \text {, }
\end{aligned}
$$

where

$$
\alpha_{2}=M, \text { and } \beta_{2}=\frac{K^{2}}{M} .
$$

It is evident from the expressions (40) to (44) that there arises a thin boundary layer of thickness of $O\left(\alpha_{2}{ }^{-1}\right)$ near lower plate of the channel. This boundary layer is called Hartmann boundary layer. The thickness of this boundary layer decreases with the increase in $M^{2}$. Outside boundary layer region i.e. when $\eta>1 / \alpha_{2}$, we obtain from (40) to (43) as

$$
\begin{aligned}
& u \approx 1 / 2, w \approx-K^{2} / 2 M, \\
& h_{x} \approx 1 / 2 M, h_{z} \approx K^{2}(2 \eta-1) / 2 M^{2} .
\end{aligned}
$$

Expressions (45) and (46) reveal that in a certain core given by $\alpha_{2}>1 / M$ i.e. outside the boundary layer region fluid flows in both the primary and secondary flow directions and primary velocity $u$ is unaffected by both rotation and magnetic field. Both the 
primary and secondary induced magnetic fields persist and secondary induced magnetic field $h_{z}$ varies linearly with the channel width variable $\eta$.

\section{Heat Transfer Characteristics}

We shall now discuss heat transfer characteristics of steady hydromagnetic Couette flow of a viscous incompressible electrically conducting fluid in a rotating system when the upper and lower plates of the channel are maintained at uniform temperatures $T_{1}$ and $T_{0}$ respectively, where $T_{0}<T<T_{1}, T$ being fluid temperature.

The energy equation taking viscous and Joule dissipations into account is given by

$$
\alpha^{*} \frac{d^{2} T}{d y^{2}}+\frac{v}{C_{p}}\left[\left(\frac{d u^{*}}{d y}\right)^{2}+\left(\frac{d w^{*}}{d y}\right)^{2}\right]+\frac{1}{\sigma \rho C_{p}}\left[\left(\frac{d H_{x}^{*}}{d y}\right)^{2}+\left(\frac{d H_{z}^{*}}{d y}\right)^{2}\right]=0,
$$

where $\alpha^{*}$ and $C_{p}$ are thermal diffusivity and specific heat at constant pressure respectively.

Boundary conditions for temperature field are

$$
T=T_{0} \text { at } y=0 \text { and } T=T_{1} \text { at } y=d .
$$

Using the non-dimensional variables defined in (12) equation (47), in non-dimensional form, becomes

$$
\frac{d^{2} \theta}{d \eta^{2}}+P_{r} E_{r}\left[\left\{\left(\frac{d u}{d \eta}\right)^{2}+\left(\frac{d w}{d \eta}\right)^{2}\right\}+M^{2}\left\{\left(\frac{d h_{x}}{d \eta}\right)^{2}+\left(\frac{d h_{z}}{d \eta}\right)^{2}\right\}\right]=0
$$

where $\theta=\frac{T-T_{0}}{T_{1}-T_{0}}, P_{r}=\frac{v}{\alpha^{*}}$ and $E_{r}=\frac{U_{0}^{2}}{C_{p}\left(T_{1}-T_{0}\right)} \cdot \theta, P_{r}$ and $E_{r}$ are non-dimensional fluid temperature, Prandtl number and

Eckert number respectively.

Boundary conditions (48), in non-dimensional form, become

$$
\theta(0)=0 \text { and } \theta(1)=1 \text {. }
$$

Making use of the equations (23) to (25) in equation (49), the resulting differential equation subject to the boundary conditions (50) is solved numerically with the help of MATLAB software. The numerical values of the rate of heat transfer at the lower and upper plates are also computed with the help of MATLAB software.

\section{Results and Discussion}

To study the effects of magnetic field and rotation on fluid velocity and induced magnetic field numerical values of the fluid velocity and induced magnetic field, computed from the analytical solution reported in section 2 by MATLAB software, are displayed graphically versus channel width variable $\eta$ in figures 1 to 4 for various values of magnetic parameter $M^{2}$ and rotation parameter $K^{2}$. Figure 1 illustrates the influence of rotation on the primary velocity $u$ and secondary velocity $w$. It is noticed that from figure 1 that both the primary and secondary velocities increase on increasing $K^{2}$ which implies that rotation tends to accelerate fluid flow in both the primary and secondary flow directions. Figure 2 demonstrates the effect of magnetic field on both the primary and secondary fluid velocities. Figure 2 reveals that an increase in $M^{2}$ leads to an increase in the secondary velocity whereas primary velocity increases in the region near the lower plate and decreases in the region near the upper plate on increasing $M^{2}$ which implies that magnetic field tends to accelerate fluid flow in secondary flow direction and it tends to decelerate fluid flow in primary flow direction in the region near the upper plate while it has the reverse effect on the primary flow in the region near the lower plate. Figure 3 displays the influence of rotation on the primary and secondary magnetic fields. It is evident from figure 3 that primary induced magnetic field $h_{X}$ increases in the lower half of the channel whereas it decreases in the upper half of the channel on increasing $K^{2}$ whereas secondary induced magnetic field $h_{z}$ increases near the lower plate as well as near the upper plate on increasing $K^{2}$. This implies that rotation tends to enhance primary induced magnetic field in the lower half of the channel and it has reverse effect on the primary induced magnetic field in the upper half of the channel. Rotation tends to increase secondary magnetic field in the region near the lower and upper plates of the channel. Figure 4 depicts the influence of magnetic 
field on the primary and secondary induced magnetic fields. It is revealed from figure 4 that primary induced magnetic field $h_{X}$ and secondary induced magnetic field $h_{z}$ decrease on increasing $M^{2}$ which implies that magnetic field has tendency to reduce both the primary and secondary induced magnetic fields.

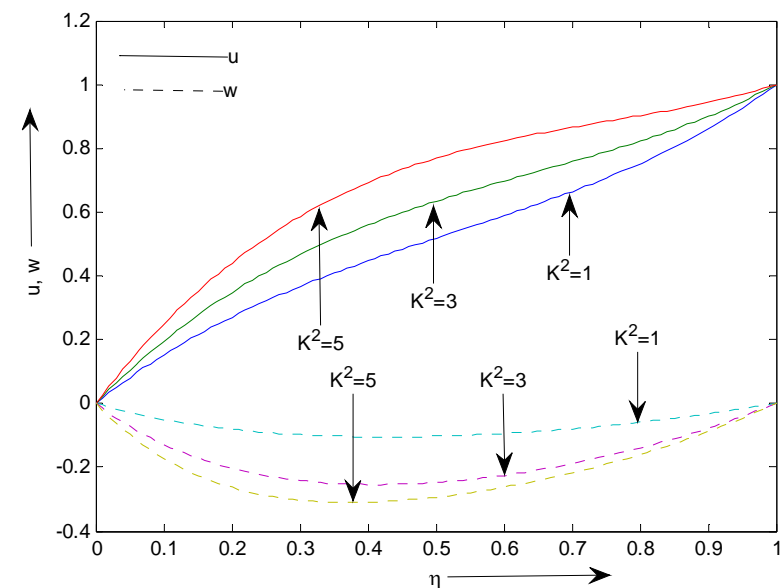

Figure 1. Velocity profiles for $M^{2}=9$

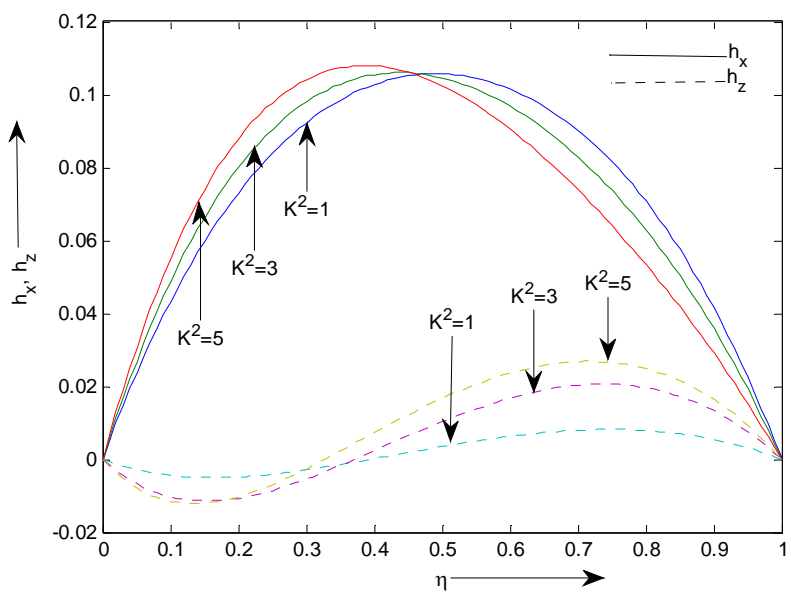

Figure 3. Magnetic field profiles for $M^{2}=9$

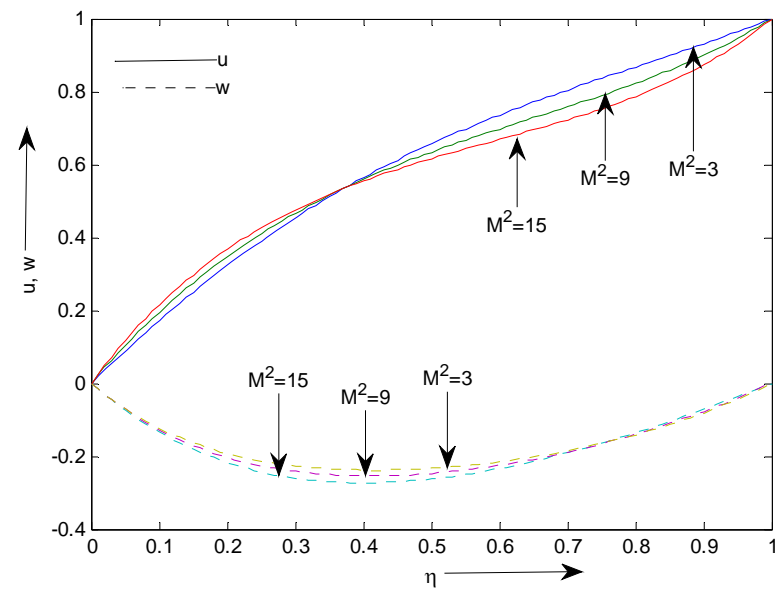

Figure 2. Velocity profiles for $K^{2}=3$

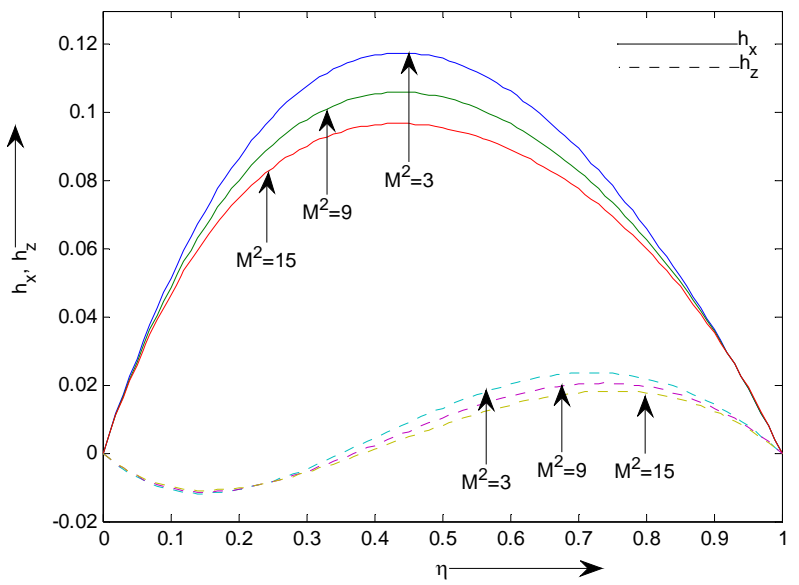

Figure 4. Magnetic field profiles for $K^{2}=3$

To study the effects of rotation and magnetic field on fluid temperature the numerical solution of energy equation computed with the help of MATLAB software is depicted graphically versus channel width variable $\eta$ in figures 5 and 6 for various values of $M^{2}$ and $K^{2}$ taking $P_{r}=7.0$ and $E_{r}=2.0$. Figure 5 displays the influence of rotation on the fluid temperature $\theta$. It is noticed from figure 5 that the fluid temperature $\theta$ increases in the regions near the upper and lower plates on increasing $K^{2}$ which implies that rotation tends to enhance fluid temperature in the regions near the upper and lower plates. Figure 6 presents the effect of magnetic field on the fluid temperature. It is evident from figure 6 that fluid temperature increases on increasing $M^{2}$ which implies that magnetic field has tendency to increase fluid temperature throughout the channel. 


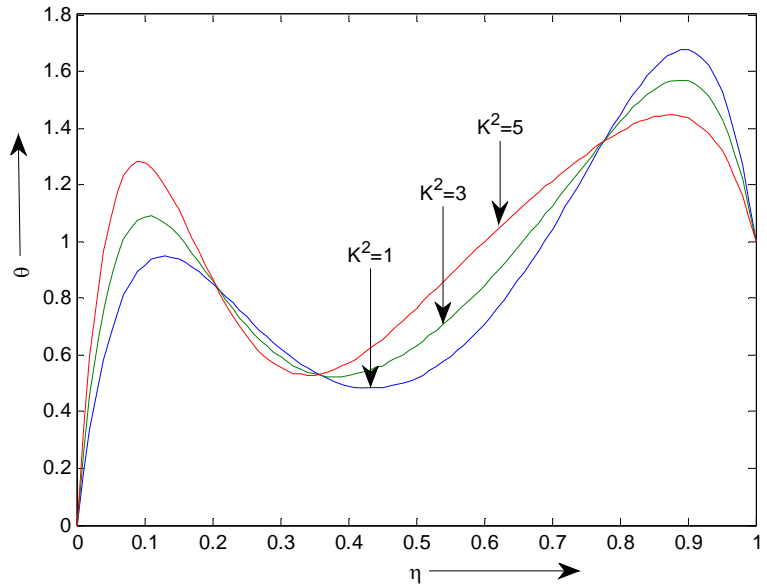

Figure 5. Fluid temperature profiles for $M^{2}=9$

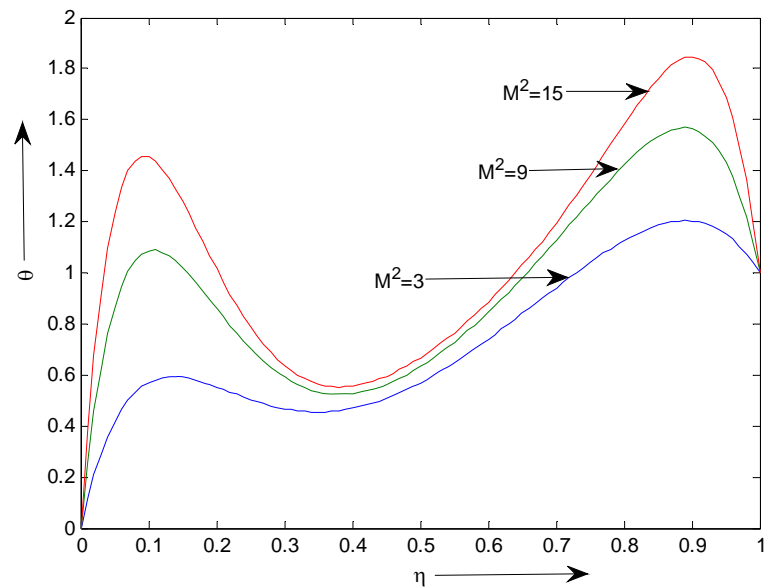

Figure 6. Fluid temperature profiles for $K^{2}=3$

The numerical values of the primary shear stress component $\tau_{X}$ and secondary shear stress component $\tau_{Z}$ at the lower and upper plates, computed from the analytical expression mentioned in section 2 by MATLAB software, are presented in tabular form in tables 1 and 2 while that of mass flow rates $Q_{X}$ and $Q_{Z}$ in the primary and secondary flow directions respectively are given in table 3 for various values of $M^{2}$ and $K^{2}$. It is evident from table 1 that primary shear stress at the lower plate i.e. $\left.\tau_{x}\right|_{\eta=0}$ and secondary shear stress at the lower plate i.e. $\left.\tau_{z}\right|_{\eta=0}$ increase on increasing $K^{2}$ where as $\left.\tau_{x}\right|_{\eta=0}$ increases and $\left.\tau_{z}\right|_{\eta=0}$ decreases on increasing $M^{2}$. This implies that rotation has tendency to enhance primary as well as secondary shear stress at the lower plate whereas magnetic field has tendency to increase primary shear stress at the lower plate and it has reverse effect on the secondary shear stress at the lower plate. It is observed from table 2 that $\left.\tau_{X}\right|_{\eta=1}$ decreases whereas $\left.\tau_{Z}\right|_{\eta=1}$ increases on increasing $\left.K^{2} \cdot \tau_{x}\right|_{\eta=1}$ and $\left.\tau_{z}\right|_{\eta=1}$ increase on increasing $M^{2}$ which implies that magnetic field tends to enhance both the primary and secondary shear stress at the upper plate and rotation has tendency to reduce primary shear stress at the upper plate and it has reverse effect on the secondary shear stress at the upper plate. It is noticed from table 3 that primary mass flow rate $Q_{X}$ and secondary mass flow rate $Q_{Z}$ increase on increasing $K^{2}$ whereas primary mass flow rate $Q_{X}$ decreases on increasing $M^{2}$ and secondary mass flow rate $Q_{z}$ decreases on increasing $M^{2}$ when $K^{2}=1$ and 3 . For $K^{2}=5$ it increases, attains a maximum value and then decreases on increasing $M^{2}$. This implies that rotation tends to enhance both the primary and secondary mass flow rates whereas magnetic field has tendency to reduce primary mass flow rate and it tends to reduce secondary mass flow rate when $K^{2} \leq 3$.

Table 1. Primary and secondary shear stress at the lower plate:

\begin{tabular}{|c|c|c|c|c|c|c|}
\hline$M^{2} \rightarrow$ & \multicolumn{3}{|c|}{$\left.\tau_{x}\right|_{\eta=0}$} & \multicolumn{3}{|c|}{$-\left.\tau_{z}\right|_{\eta=0}$} \\
\hline & 3 & 9 & 15 & 3 & 9 & 15 \\
\hline 1 & 1.3195 & 1.7312 & 2.0873 & 0.6369 & 0.6167 & 0.6023 \\
\hline 3 & 1.8179 & 2.2033 & 2.5372 & 1.6443 & 1.6173 & 1.6007 \\
\hline 5 & 2.3937 & 2.7818 & 3.1171 & 2.2537 & 2.2365 & 2.2354 \\
\hline
\end{tabular}


Table 2. . Primary and secondary shear stress at the upper plate:

\begin{tabular}{|c|c|c|c|c|c|c|}
\hline$M^{2}$ & \multicolumn{3}{|c|}{$\left.\tau_{x}\right|_{\eta=1}$} & \multicolumn{3}{|c|}{$\left.\tau_{z}\right|_{\eta=1}$} \\
\hline & 3 & 9 & 15 & 3 & 9 & 15 \\
\hline 1 & 1.1662 & 1.5894 & 1.9548 & 0.3341 & 0.3589 & 0.3767 \\
\hline 3 & 0.7387 & 1.1668 & 1.5411 & 0.7451 & 0.8496 & 0.9273 \\
\hline 5 & 0.2983 & 0.6838 & 1.0316 & 0.7845 & 0.9749 & 1.1246 \\
\hline
\end{tabular}

Table 3. Primary and secondary mass flow rates:

\begin{tabular}{|c|c|c|c|c|c|c|}
\hline \multirow{2}{*}{$K^{2} \downarrow$} & \multicolumn{3}{|c|}{$Q_{x}$} & \multicolumn{3}{|c|}{$-Q_{z}$} \\
\hline & 3 & 9 & 15 & 3 & 9 & 15 \\
\hline 1 & 0.5145 & 0.5122 & 0.5105 & 0.0767 & 0.0709 & 0.0662 \\
\hline 3 & 0.6018 & 0.5889 & 0.5787 & 0.1799 & 0.1727 & 0.1660 \\
\hline 5 & 0.6962 & 0.6789 & 0.6640 & 0.2095 & 0.2098 & 0.2085 \\
\hline
\end{tabular}

The numerical values of rate of heat transfer at the lower and upper plates are computed with the help of MATLAB software and are presented in table 4 for various value of $K^{2}$ and $M^{2}$ taking $P_{r}=7.0$ and $E_{r}=2.0$. It is found from table 4 that rate of heat transfer at the lower plate i.e. $\left.\frac{d \theta}{d \eta}\right|_{\eta=0}$ increases on increasing $K^{2}$ whereas rate of heat transfer at the upper plate i.e. $\left.\frac{d \theta}{d \eta}\right|_{\eta=1}$ decreases on increasing $K^{2}$. Both $\left.\frac{d \theta}{d \eta}\right|_{\eta=0}$ and $\left.\frac{d \theta}{d \eta}\right|_{\eta=1}$ increase on increasing $M^{2}$ which implies that rotation tends to enhance rate of heat transfer at the lower plate and it has reverse effect on the rate of heat transfer on the upper plate. Magnetic field has tendency to enhance the rate of heat transfer at both the upper and lower plates.

Table 4. Rate of heat transfer at the lower and upper plates:

\begin{tabular}{|c|c|c|c|c|c|c|}
\hline$M^{2} \rightarrow$ & & $\left(\frac{d \theta}{d \eta}\right)_{\eta=0}$ & & & $-\left(\frac{d \theta}{d \eta}\right)_{\eta=}$ & \\
\hline & 3 & 9 & 15 & 3 & 9 & 15 \\
\hline 1 & 8.5768 & 19.7156 & 30.6782 & 5.7642 & 16.0090 & 26.3293 \\
\hline 3 & 12.0455 & 27.3610 & 41.5914 & 4.3464 & 12.9220 & 21.9689 \\
\hline 5 & 15.8003 & 36.3406 & 55.2513 & 2.8140 & 9.3003 & 16.5157 \\
\hline
\end{tabular}

\section{Conclusion}

This study presents a theoretical investigation of steady hydromagnetic Couette flow of class-II in a rotating system. The significant findings are summarized below 
(i) Rotation tends to accelerate fluid flow in both the primary and secondary flow directions.

(ii) Magnetic field tends to accelerate fluid flow in secondary flow direction and it tends to decelerate fluid flow in primary flow direction in the region near the upper plate where it has reverse effect on the primary flow in the region near the lower plate.

(iii) Rotation tends to enhance primary induced magnetic field in the lower half of the channel and it has the reverse effect on the primary induced magnetic field in the upper half of the channel. Rotation tends to increase secondary induced magnetic field in the regions near the lower and upper plates of the channel.

(iv) Magnetic field has tendency to reduce both the primary and secondary induced magnetic fields.

(v) Rotation tends to enhance fluid temperature in the regions near the lower and upper plates of the channel.

(vi) Magnetic field has tendency to increase fluid temperature throughout the channel.

(vii) Rotation has tendency to enhance primary as well as the secondary shear stress at the lower plate and magnetic field has tendency to increase the primary shear stress at the lower plate and it has reverse effect on the secondary shear stress at the lower plate.

(viii) Magnetic field tends to enhance both the primary and secondary shear stress at the upper plate and rotation has tendency to reduce primary shear stress at the upper plate and it has the reverse effect on the secondary shear stress at the upper plate.

(ix) Rotation tends to enhance both the primary and secondary mass flow rates whereas the magnetic field has tendency to reduce primary mass flow rate.

(x) Rotation tends to enhance rate of heat transfer at the lower plate and it has reverse effect on the rate of heat transfer at the upper plate.

(xi) Magnetic field has tendency to enhance rate of heat transfer at both the lower and upper plates.

\section{References}

Das, B. K., Guria, M. and Jana, R. N., 2008, Unsteady Couette flow in rotating system, Meccanica, Vol. 43, pp. 517.

Ganapathy, R., 1994, A note on oscillatory Couette flow in a rotating system, ASME J. Appl. Mech., Vol. 61, pp. 208.

Hayat, T., Nadeem, S. and Asghar, S., 2004a, Hydromagnetic Couette flow of an Oldroyd -B fluid in rotating system, Int. J. Engng. Sci., Vol. 42, pp. 65.

Hayat, T., Nadeem, S., Siddiqui, A. M. and Asghar, S., 2004b, An oscillatory hydrmagnetic non-Newtonian flow in a rotating system, Appl. Math. Lett., Vol. 17, pp. 609.

Hayat, T., Wang, Y. and Hutter, K., 2004c, Hall effects on unsteady hydromagnetic oscillatory flow of a second-grade fluid, Int. J. Nonlinear Mech., Vol. 39, pp. 1027.

Jana, R. N., Datta, N. and Majumdar, B. S., 1977, Magnetohydrodynamic Couette flow and heat transfer in rotating system, J. Phys. Soc. Japan, Vol. 42, pp. 1034.

Jana, R. N. and Datta, N., 1980, Hall effects on MHD Couette flow in a rotating system, Czech. J. Phys., Vol. B30, pp. 659.

Kumar, A., Seth, G. S. and Talib, A., 2007, Hydromagnetic Couette flow in a rotating system with Hall effects, Acta Ciencia Indica, Vol. 33M, pp. 937.

Lenzo, C. S., Dauzvardis, P. V. and Hantman, R. G., 1978, An experimental investigation of rotating-drum separators for liquidmetal MHD applications, $17^{\text {th }}$ Symposium on the Engineering Aspects of Magnetohydrodynamics, Stanford, Calif., March 2729.

Mandal, G. and Mandal, K. K., 1983, Effects of Hall current on MHD Couette flow between thick arbitrarily conducting plates in a rotating system, J. Phys. Soc. Japan, Vol. 52, pp. 470.

Mandal, G., Mandal, K.K. and Choudhury, G., 1982, On combined effects of Coriolis force and Hall current on steady MHD Couette flow and heat transfer, J. Phys. Soc. Japan, Vol. 51, pp. 2010.

Majumder, B. S., 1991, An exact solution of oscillatory Couette flow in a rotating system, ASME J. Appl. Mech., Vol. 8, pp. 1104.

Michiyoshi, I. and Numano, M., 1967, Performance characteristics of a vortex-type MHD power generator-II, Plasma Physics, Vol. 9, pp. 549.

Seth, G. S. and Maiti, M. K., 1982, MHD Couette flow and heat transfer in a rotating system, Ind. J. Pure Appl. Math., Vol. 13, pp. 931.

Seth, G. S. and Ahmad, N., 1985, Effect of Hall current on MHD Couette flow and heat transfer in a rotating system, J. ISTAM, Vol.30, pp. 931.

Seth, G. S., Nandkeolyar, R. and Ansari, Md. S., 2009, Hall effects on oscillatory hydromagnetic Couette flow in a rotating system, Int. J. Acad. Res., Vol. 1, pp. 6.

Singh, K, D., 2000, An oscillatory hydromagnetic Couette flow in a rotating system, ZAMM, Vol. 80, pp. 429.

Thiagarajan, V. and Rohatgi, V. K., 1977, Analysis of a collisional plasma rotating inside a cylindrical annulus, Energy Conversion, Vol. 17, pp. 73. 


\section{Biographical notes}

Dr. G. S. Seth is a Professor in the Department of Applied Mathematics, Indian School of Mines, Dhanbad, India. He received his Ph. D. in the Mathematics from Indian Institute of Technology, Kharagpur, India. He has more than twenty nine years of experience of teaching and research. His current area of research studies includes Fluid dynamics, Magnetohydrodynamics and heat transfer. He was visiting Assistant Professor at University of Aden, Republic of Yemen during the period September 01, 1991 to August 31, 1993. He has published more than forty eight research papers in national/International journals of repute.

J. K. Singh received M. Sc. Degree in Mathematics from D. D. U. Gorakhpur University, Gorakhpur, India in the year 2004. He joined the Department of Applied Mathematics, Indian School of Mines, Dhanbad, India as Project Fellow in the year 2008. After one year he has joined the same institute as CSIR-UGC (NET) Junior Research Fellow to do research work leading Ph. D. degree on the research topic entitled "A theoretical study of the problems of Magnetohydrdynamic flow in a rotating system. He has two and half years of research experience.

Received September 2010

Accepted June 2011

Final acceptance in revised form June 2011 